\title{
QUOTIENT RINGS, CHAIN CONDITIONS AND INJECTIVE RING ENDOMORPHISMS
}

\author{
by J. C. WILKINSON
}

(Received 15 December, 1987)

1. Introduction. In this paper, the situation we shall be concerned with is that a ring $R$, with a ring monomorphism $\alpha: R \rightarrow R$, which will not be assumed to be surjective.

Much work has been done on the skew polynomial ring $R[x, \alpha]$ and the skew Laurent polynomial ring $R\left[x, x^{-1}, \alpha\right]$, where $\alpha$ is an automorphism-see [3] for example. However, the fact that $\alpha$ is not surjective renders the study of these objects much more difficult.

It is with this in mind that $\mathrm{D}$. A. Jordan [4] constructs a minimal overring $A(R, \alpha)$ to which $\alpha$ extends as an automorphism $\bar{\alpha}$ say. Using the fact that $A(R, \alpha)\left[x, x^{-1}, \bar{\alpha}\right]$ coincides with $R\left[x, x^{-1}, \alpha\right]$ (see [4]), it is clear that existing results for the case where $\alpha$ is an automorphism can be used in the non-surjective case, provided we can handle the relationship between $R$ and $A(R, \alpha)$. This paper studies that relationship, with particular regard to chain conditions and quotient rings for $A(R, \alpha)$.

The paper is divided into three main sections, the first of which deals with conditions on a left Noetherian ring which are equivalent to $A(R, \alpha)$ being a left order in a left Artinian ring. The second section answers in the negative a question raised by Jordan in [4], where he asks whether $R$ having left Krull dimension 1 is sufficient to ensure that $A(R, \alpha)$ has left Krull dimension. The final section presents an example which shows that it is possible for $R$ to have acc on annihilator left ideals, but for this condition to fail in $A(R, \alpha)$.

2. Preliminaries. The purpose of this section is to present the relevant definitions and results concerning $A(R, \alpha)$. These come mainly from [4], and deal with the relationship between the left ideal structure of $A(R, \alpha)$ and the left ideal structure of $R$.

All rings are assumed to have unity, and it will be assumed that all monomorphisms $\alpha: R \rightarrow R$ satisfy $\alpha(1)=1$. To say that $I \subseteq R$ is an ideal will mean that it is both a left ideal and a right ideal-a similar interpretation will be placed on the words Artinian, Noetherian, and so on. The nilpotent radical $N(R)$ of a ring $R$ will be taken to be the sum of all the nilpotent left ideals of $R$, and if $S$ is a subset of $R$ then $C_{R}(S)$ will denote all the elements of $R$ which are regular modulo $S$. in [4].

We begin by defining the ring $A(R, \alpha)$. A more detailed construction may be found

Definition 2.1 [4]. Let $R$ be a ring, $\alpha: R \rightarrow R$ a ring monomorphism, and let $R\left[x, x^{-1}, \alpha\right]$ be the skew Laurent polynomial ring, having as elements finite sums of elements of the form $x^{-j} r x^{i}$, where $i, j \geqq 0$ and $r \in R$.

Then $A(R, \alpha)$ is the subring $\left\{x^{-i} r x^{i} \mid r \in R, i \geqq 0\right\}$ of $R\left[x, x^{-1}, \alpha\right]$.

Glasgow Math. J. 31 (1989) 173-181. 
Remark. It can be shown (see [4]) that $A(R, \alpha)$ is, up to isomorphism, the minimal overring of $R$ to which $\alpha$ extends as an automorphism. The action of $\alpha$ on $A(R, \alpha)$ is defined by $\alpha\left(x^{-i} r x^{i}\right)=x^{-i} \alpha(r) x^{i}$, and no confusion should arise from the fact that $\alpha$ denotes both the original monomorphism on $R$ and the automorphism on $A(R, \alpha)$.

Definition 2.2 [4]. Let $\alpha: R \rightarrow R$ be a ring monomorphism. Then a left ideal $I$ of $R$ is said to be closed if $\bigcup_{n \geq 0} \alpha^{-n}\left(R \alpha^{n}(I)\right) \subseteq I$.

DEFINITION 2.3 [4]. A sequence $\left(I_{i}\right)_{i \geqq 0}$ of subsets of $R$ such that, for all $i \geqq 0$, $\alpha^{-1}\left(I_{i+1}\right)=I_{i}$ is called an $\alpha$-sequence.

Remark. It is easily shown that, given an $\alpha$-sequence $\left(I_{i}\right)_{i \geq 0}$ of left ideals of $R, I_{i}$ is closed for each $i \geqq 0$.

DEFINITION 2.4 [4]. Let $\left(I_{i}\right)_{i \gtrless 0}$ and $\left(J_{i}\right)_{i \gtrless 0}$ be $\alpha$-sequences of closed left ideals of $R$. Then define a relation $\leqq$ on the set of $\alpha$-sequences of closed left ideals of $R$ by putting $\left(I_{i}\right)_{i \geq 0} \leqq\left(J_{i}\right)_{i \geqq 0}$ if and only if $I_{i} \subseteq J_{i}$ for all $i \geqq 0$.

It is clear that $\leqq$ defines a partial ordering on the set of all $\alpha$-sequences of closed left ideals of $R$. The significance of these three definitions is made precise by the following theorem.

THEOREM 2.5 [4]. There exists an order-preserving bijection $\Gamma$ from the lattice of left ideals of $A(R, \alpha)$ to the partially ordered set of $\alpha$-sequences of closed left ideals of $R$ given by

$$
\Gamma(I)=\left(I_{i}\right)_{i \geqq 0}, \text { where } I_{i}=\left\{r \in R \mid x^{-i} r x^{i} \in I\right\} .
$$

The inverse map $\Delta$ is given by

$$
\Delta\left(I_{i}\right)_{i \succeq 0}=\bigcup_{i \geq 0} x^{-i} I_{i} x^{i}
$$

and is also order-preserving.

Proof. Theorem 4.7 of [4].

Among the consequences of Theorem 2.5 are the following results.

Theorem 2.6. If $R$ is left Artinian then $A(R, \alpha)$ is also left Artinian.

Proof. See [4, Corollary 5.3].

THEOREM 2.7. If $R$ is a semiprime left Goldie ring then $A(R, \alpha)$ is a semiprime left Goldie ring.

Proof. Corollary 7.4 of [4].

DefintTion 2.8. Let $R$ be a ring and $\alpha: R \rightarrow R$ a monomorphism. Then a left, right, or two sided ideal $I$ is said to be $\alpha$-invariant if $\alpha(I) \subseteq I$. It is said to be $\alpha$-stable if $I$ is $\alpha$-invariant and $\alpha^{-1}(I) \subseteq l$. 
The above definitions often prove useful when dealing with certain ideals of $A(R, \alpha)$. In particular, it is easy to see that the nilpotent radical of a ring is stable under any automorphism of that ring-so $N(A(R, \alpha))$ is stable under the automorphism $\alpha: A(R, \alpha) \rightarrow A(R, \alpha)$.

Proposition 2.9. Let $I$ be an $\alpha$-stable left ideal of $A(R, \alpha)$, with corresponding $\alpha$-sequence $\Gamma(I)=\left(I_{i}\right)_{i \geqq 0}$. Then $I_{i}=I_{j}$ for all $i, j \geqq 0$.

Proof. Let $i \geqslant 0$ and let $r \in I_{i}$. Then $x^{-i} r x^{i} \in I$, and since $I$ is an $\alpha$-stable left ideal of $A(R, \alpha), \alpha^{i}\left(x^{-i} r x^{i}\right) \in I$, i.e. $r \in I_{0}$.

Now, if $r \in I_{0}$ then $r \in I$ and, since $I$ is $\alpha$-stable, $\alpha^{-i}(r) \in I$, i.e. $x^{-i} r x^{i} \in I$, or $r \in I_{i}$.

3. Artinian quotient rings. The aim of this section is to obtain necessary and sufficient conditions on a left Noetherian ring $R$ so that $A(R, \alpha)$ is a left order in a left Artinian ring. In addition to $R$ being left Noetherian, it will be assumed that the nilpotent radical $N(R)$ of $R$ is $\alpha$-invariant-this assumption is not very restrictive, as there are no known examples of a left Noetherian ring whose nilpotent radical is not $\alpha$-invariant. Moreover, it has been shown by Dean [1] that if $R$ is left Noetherian with acc on right annihilators then $N(R)$ is invariant under any monomorphism $\alpha: R \rightarrow R$.

It is important to note that $A(R, \alpha)$ is not assumed to be left Noetherian, and the proof of the main result relies on a non-Noetherian version of Small's theorem. Before presenting this variation of Small's theorem, we recall the definition of reduced rank for a left $R$-module.

Defintrion 3.1. Let $R$ be a ring, $M$ a left $R$-module, and $Z(M)$ the singular submodule of $M$.

(i) If $R$ is a semiprime left Goldie ring then the reduced rank $\rho(M)$ of $M$ is defined to be the Goldie dimension of $M / Z(M)$.

(ii) If $R$ is such that the nilpotent radical $N$ of $R$ is nilpotent and $R / N$ is a left Goldie ring then the reduced rank $\rho(M)$ of $M$ is given by

$$
\rho_{R}(M)=\sum_{i=0}^{k-1} \rho_{R / N}\left(\frac{N^{i} M}{N^{i+1} M}\right),
$$

where $N^{k}=0, N^{0}=R$, and the reduced ranks on the right are calculated as in (i).

THEOREM 3.2. Let $R$ be a ring with nilpotent radical $N$. Then $R$ has a left Artinian left quotient ring if and only if:

(i) $N$ is nilpotent;

(ii) $R / N$ is a left Goldie ring;

(iii) $\rho_{R}\left({ }_{R} R\right)$ is finite;

(iv) $C_{R}(N)=C_{R}(0)$.

Proof. This is Theorem 3 of [6].

We shall prove that conditions (i), (ii) and (iii) hold automatically for $A(R, \alpha)$ in the case where $R$ is left Noetherian with $\alpha$-invariant nilpotent radical. 
LemmA 3.3. Let $R$ be a left Noetherian ring with nilpotent radical $N(R)$ such that $\alpha(N(R)) \subseteq N(R)$. Then

(i) the nilpotent radical $N$ of $A(R, \alpha)$ is given by $N=\bigcup_{i \geqq 0} x^{-i} N(R) x^{i}$,

(ii) $N$ is nilpotent.

Proof. (i) Denote $\bigcup_{i \geqq 0} x^{-i} N(R) x^{i}$ by $I$. It is straightforward to show that $I$ is an ideal of $A(R, \alpha)$ and, since $R$ is left Noetherian, $N(R)$ is nilpotent-say $N(R)^{k}=0$. Then

Let $x^{-i_{j}} a_{j} x^{i_{j}} \in I$, where $a_{j} \in N(R)$ and $i_{j} \geqq 0$ for $j=1, \ldots, k, i=\max \left\{i_{1}, \ldots, i_{k}\right\}$.

$$
\begin{aligned}
\prod_{j=1}^{k} x^{-i_{j}} a_{j} x^{i_{j}} & =\prod_{j=1}^{k} x^{-i} \alpha^{i-i_{j}}\left(a_{j}\right) x^{i} \\
& =x^{-i}\left(\prod_{j=1}^{k} \alpha^{i-i_{j}}\left(a_{j}\right)\right) x^{i} \\
& =0
\end{aligned}
$$

since $N(R)$ is $\alpha$-invariant. Thus $I^{k}=0$ and $I \subseteq N$.

Now, since $N$ is an $\alpha$-stable ideal of $A(R, \alpha)$, Proposition 2.9 gives $N_{i}=N_{0}=N \cap R$, where $\Gamma(N)=\left(N_{i}\right)_{i \geqq 0}$. But $N \cap R$ is a nilpotent ideal of $R$; so $N \cap R \subseteq N(R)$ and, by Theorem 2.5,

$$
N=\bigcup_{i \geqq 0} x^{-i}(N \cap R) x^{i} \subseteq I
$$

(ii) This is immediate from (i).

Lemma 3.4. Let $R$ be a left Noetherian ring such that $N(R)$ is $\alpha$-invariant. Then $A(R, \alpha) / N$ is a semiprime left Goldie ring.

Proof. Let $k$ be such that $N(R)^{k}=0$ and let $r \in \alpha^{-1}(N(R))$. Then $\alpha\left(r^{k}\right)=\alpha(r)^{k} \in$ $N(R)^{k}=0$, and $\alpha^{-1}(N(R))$ is a nilpotent ideal of $R$. Thus $\alpha^{-1}(N(R)) \subseteq N(R)$ ); so that $N(R)$ is $\alpha$-stable.

Thus it is possible to define a ring monomorphism $\bar{\alpha}: R / N(R) \rightarrow R / N(R)$ by

$$
\bar{\alpha}(r+N(R))=\alpha(r)+N(R) .
$$

Now, it can be shown that $\psi: A(R / N(R), \bar{\alpha}) \rightarrow A(R, \alpha) / N$ defined by

$$
\psi\left(x^{-i}(r+N(R)) x^{i}\right)=x^{-i} r x^{i}+N
$$

is a well-defined ring homomorphism. In fact, $\psi$ can be seen to be an isomorphism; so that $A(R, \alpha) / N$ is isomorphic to $A(R / N(R), \bar{\alpha})$. But $R / N(R)$ is a semiprime left Goldie ring; so, by Theorem $2.7, A(R / N(R), \bar{\alpha})$, and hence $A(R, \alpha) / N$, are also semiprime left Goldie.

Lemma 3.5. Let $R$ be a left Noetherian ring and let $J$ be an $\alpha$-stable left ideal of $A(R, \alpha)$. Then $A(R, \alpha) / J$ has finite left Goldie dimension. 
Proof. Assume that $A(R, \alpha) / J$ does not have finite left Goldie dimension. Then there exists a sequence $\left(K_{i}\right)_{i \geq 0}$ of left ideals of $A(R, \alpha)$ such that $J \varsubsetneqq K_{i}$ and the sum $\sum_{i=0}^{\infty} K_{i} / J$ is direct. If $\left(K_{i j}\right)_{j \geq 0}$ denotes the $\alpha$-sequence $\Gamma\left(K_{i}\right)$ and $\left(J_{j}\right)_{j \geq 0}$ denotes the $\alpha$-sequence $\Gamma(J)$ then, by Theorem $2.5, J_{j} \subseteq K_{i j}$ for all $i, j \geqq 0$. It is now claimed that, for each $j \geqq 0$, the sum $\sum_{i=0}^{\infty} K_{i j} / J_{j}$ is direct.

Indeed, for $j \geqq 0$, let $r_{i} \in K_{i j}$ (for $i=0, \ldots, p$ ) be such that $\sum_{i=0}^{p} r_{i}+J_{j}=0$. Then $\sum_{i=0}^{p} r_{i} \in J_{j}$; so that $x^{-j} \sum_{i=0}^{p} r_{i} x^{j} \in J$, or $\sum_{i=0}^{p} x^{-j} r_{i} x^{j} \in J$. Since $x^{-j} r_{i} x^{j} \in K_{i}$, directness of the sum $\sum_{i=0}^{\infty} K_{i} / J$ means that $x^{-j} r_{i} x^{j} \in J$, i.e. $r_{i} \in J_{j}$, for each $i=1, \ldots, p$ and this proves directness of the sum $\sum_{i=0}^{\infty} K_{i j} / J_{j}$.

Now, since $J \subsetneq K_{i}$ for each $i \geqq 0$, there exists $l \geqq 0$ with $J_{l} \subsetneq K_{i l}$. Furthermore, if $r \in K_{i l}-J_{l}$ then $x^{-l} r x^{l} \in K_{i}-J$. Therefore, for any $k \geqq 0, x^{-(l+k)} \alpha^{k}(r) x^{l+k} \in K_{i}-J$; so that $\alpha^{k}(r) \in K_{i, l+k}-J_{l+k}$. Thus, for each $i \geqq 0$, there exists $l_{0} \geqq 0$ such that, for all $l \geqq l_{0}$, $K_{i l} / J_{l} \neq 0$. Therefore, there exists $j_{0} \geqq 0$ such that $K_{0, j_{0}} / J_{j_{0}} \neq 0$.

By the above argument, there exists $j_{1} \geqq j_{0}$ with $K_{1, j_{1}} / J_{j_{1}} \neq 0, K_{0, j_{1}} / J_{j_{1}} \neq 0$ and the sum $K_{0, j_{1}} / J_{j_{1}}+K_{1, j_{1}} / J_{j_{1}}$ direct.

The procedure can be repeated indefinitely to yield, for any $n \geqq 0$, a direct sum

$$
\frac{K_{0, j_{n}}}{J_{j_{n}}} \oplus \frac{K_{1, j_{n}}}{J_{j_{n}}} \oplus \ldots \oplus \frac{K_{n, j_{n}}}{J_{j_{n}}}
$$

of non-zero submodules of $R / J_{j_{n}}$. But $J$ is an $\alpha$-stable left ideal of $A(R, \alpha)$; so by Proposition 2.9, $J_{j}=J_{0}=J \cap R$ for all $j \geqq 0$. This means that, given any $n \geqq 0$, there exists a direct sum

$$
\frac{K_{0, j_{n}}}{J \cap R} \oplus \ldots \oplus \frac{K_{n, j_{n}}}{J \cap R}
$$

of non-zero submodules of $R /(J \cap R)$. This is impossible since $R$ is left Noetherian, and $R /(J \cap R)$, therefore, has finite Goldie dimension.

Lemma 3.6. Let $R$ be a left Noetherian ring with $N(R) \alpha$-invariant. Then $\rho(A(R, \alpha))<\infty$, where $\rho$ denotes the reduced rank of a left $A(R, \alpha)$-module.

Proof. By Proposition 3.3, the nilpotent radical $N$ of $A(R, \alpha)$ is nilpotent, of index $k$ say. By Lemma 3.4, $A(R, \alpha) / N$ is a semiprime left Goldie ring. Then, from Definition 3.1 , the reduced rank of $A(R, \alpha)$ is given by

$$
\rho(A(R, \alpha))=\sum_{i=1}^{k-1} \rho_{A(R, \alpha) / N}\left(\frac{N^{i}}{N^{i+1}}\right) .
$$


It is therefore sufficient to show that $\rho_{A(R, \alpha) / N}\left(\frac{N^{i}}{N^{i+1}}\right)$ is finite, for each $i=0, \ldots, k-1$.

Consider the singular submodule $Z\left(N^{i} / N^{i+1}\right)$ of the $A(R, \alpha) / N$-module $N^{i} / N^{i+1}$, and denote the set $C_{A(R, \alpha)}(N)$ by $C(N)$. By definition,

$$
\begin{aligned}
Z\left(\frac{N^{i}}{N^{i+1}}\right) & =\left\{r+N^{i+1} \mid c r \in N^{i+1} \text { for some } c \in C(N)\right\} \\
& =\frac{A_{i}}{N^{i+1}}
\end{aligned}
$$

where $A_{i}=\left\{r \in N^{i} \mid c r \in N^{i+1}\right.$ for some $\left.c \in C(N)\right\}$. Using the fact that $N$ is $\alpha$-stable, it can be shown that $\alpha(C(N))=C(N)$ and then that $A_{i}$ is an $\alpha$-stable left ideal of $A(R, \alpha)$. By Lemma 3.5, $N^{i} / A_{i}$ has finite Goldie dimension for each $i=0, \ldots, k-1$. Thus,

$$
\begin{aligned}
\rho_{A(R, \alpha) / N}\left(\frac{N^{i}}{N^{i+1}}\right) & =\operatorname{dim} \frac{N^{i} / N^{i+1}}{Z\left(N^{i} / N^{i+1}\right)} \\
& =\operatorname{dim} \frac{N^{i} / N^{i+1}}{A_{i} / N^{i+1}} \\
& =\operatorname{dim} N^{i} / A_{i}<\infty
\end{aligned}
$$

for each $i=0, \ldots, k-1$.

Notation. Denote by ' $C_{\alpha}(0)$ the set $\left\{r \in R \mid \alpha^{n}(r) \in{ }^{\prime} C_{R}(0)\right.$ for all $\left.n \geqq 0\right\}$, by $C_{\alpha}^{\prime}(0)$ the set $\left\{r \in R \mid \alpha^{n}(r) \in C_{R}^{\prime}(0)\right.$ for all $\left.n \geqq 0\right\}$ and by $C_{\alpha}(0)$ the set ${ }^{\prime} C_{\alpha}(0) \cap C_{\alpha}^{\prime}(0)$. Note that $C_{R}^{\prime}(0)$ and ' $C_{R}(0)$ refer to the right regular and left regular elements of $R$ respectively.

Also, the sets $C_{A(R, \alpha)}(0)$ and $C_{A(R, \alpha)}(N)$ will be denoted by $C(0)$ and $C(N)$ respectively.

We are now in a position to prove the main result of this section.

THEOREM 3.7. Let $R$ be a left Noetherian ring such that $N(R)$ is $\alpha$-invariant. Then $A(R, \alpha)$ has a left Artinian left quotient ring if and only if $C_{\alpha}(0)=C_{R}(N(R))$.

Proof. First note that, by Lemma 3.3, the nilpotent radical $N$ of $A(R, \alpha)$ is nilpotent; by Lemma 3.4, $A(R, \alpha) / N$ is a left Goldie ring and, by Lemma 3.6, $A(R, \alpha)$ has finite reduced rank, as a left $A(R, \alpha)$-module. By Theorem 3.2 , it is only necessary to show that $C_{\alpha}(0)=C_{R}(N(R))$ if and only if $C(0)=C(N)$.

As in the proof of Lemma 3.4, $N(R)$ is $\alpha$-stable; so it is possible to define a monomorphism $\bar{\alpha}: R / N(R) \rightarrow R / N(R)$ by $\bar{\alpha}(r+N(R))=\alpha(r)+N(R)$. But $R / N(R)$ is a semiprime left Noetherian ring; so, by Goldie's theorem and Proposition 2.4 of [3], $\bar{\alpha}\left(C_{R / N(R)}(0)\right) \subseteq C_{R / N(R)}(0)$ or $\alpha\left(C_{R}(N(R)) \subseteq C_{R}(N(R))\right.$.

Now let $x^{-i} r x^{i} \in C(N)$ and let $s \in R$ be such that $r s \in N(R)$. Then $x^{-i} r s x^{i}=$ 
$\left(x^{-i} r x^{i}\right)\left(x^{-i} s x^{i}\right) \in \bigcup_{j \succeq 0} x^{-i} N(R) x^{j}=N$ by Lemma 3.3. Therefore $x^{-i} s x^{i} \in N$ and $s \in N$ since $N$ is $\alpha$-stable. Thus $s \in N \cap R \subseteq N(R)$, and $r \in C_{R}^{\prime}(N(R))$. A similar argument on the left yields that $r \in C_{R}(N(R))$, and so $C(N) \subseteq \bigcup_{i \geqq 0} x^{-i} C_{R}(N(R)) x^{i}$.

On the other hand, let $r \in C_{R}(N(R))$, let $i \geqq 0$ and let $s \in R, j \geqq 0$ such that $\left(x^{-i} r x^{i}\right)\left(x^{-j} s x^{j}\right) \in N$. Then $x^{-(i+j)} \alpha^{j}(r) \alpha^{i}(s) x^{i+j} \in N$ and, since $N$ is $\alpha$-stable, $\alpha^{j}(r) \alpha^{i}(s) \in$ $N \cap R \subseteq N(R)$. Since $\alpha\left(C_{R}(N(R))\right) \subseteq C_{R}(N(R)), \alpha^{j}(r) \in C_{R}(N(R))$; so that $\alpha^{i}(s) \in N(R)$, and $x^{-(i+j)} \alpha^{i}(s) x^{i+j} \in N$ (by Lemma 3.3). Thus $x^{-j} s x^{j} \in N$ and $x^{-i} r x^{i} \in C^{\prime}(N)$. Similarly, it can be shown that $x^{-i} r x^{i} \in \in^{\prime} C(N)$; whence $\bigcup_{i \geq 0} x^{-i} C_{R}(N(R)) x^{i}=C(N)$.

By Proposition 3.1 of [4], $\bigcup_{i \geq 0} x^{-i} C_{\alpha}(0) x^{i}=C(0)$, and it is now routine to prove that $C(0)=C(N)$ if and only if $C_{\alpha}(0)=C_{R}(N(R))$. By Theorem 3.2, $A(R, \alpha)$ has a left Artinian left quotient ring if and only if $C_{\alpha}(0)=C_{R}(N(R))$.

4. Krull dimension. We now turn away from the question of left Artinian left quotient rings to consider the effect on chain conditions as we pass from $R$ to $A(R, \alpha)$. The first of these chain conditions is Krull dimension, as defined in [2].

In his paper [4], Jordan shows that it is possible to have a ring $R$ of Krull dimension 2 , and a monomorphism $\alpha: R \rightarrow R$ such that $A(R, \alpha)$ does not have Krull dimension. However, he also shows (see Theorem 2.6) that if $R$ has left Krull dimension zero then so does $A(R, \alpha)$. Thus, the question arises as to what happens when $K \operatorname{dim}_{R} R=1$. The following example settles this question by providing a commutative ring $R$ with Krull dimension 1 and a monomorphism $\alpha: R \rightarrow R$ such that $A(R, \alpha)$ does not have Krull dimension.

EXAmple 4.1. Let $R$ be the polynomial ring $K[y]$ over the field $K$, and let $\alpha: R \rightarrow R$ be the $K$-monomorphism such that $\alpha(y)=y^{2}$. Note that $R$ is a commutative, Noetherian domain of Krull dimension 1. $n \in \mathbb{N}$,

With $\left\langle y^{n}\right\rangle$ denoting the ideal of $R$ generated by $y^{n}$, it is easily seen that, for any

$$
\alpha^{-1}\left(\left\langle y^{n}\right\rangle\right)= \begin{cases}\left\langle y^{n / 2}\right\rangle & \text { for } n \text { even, } \\ \left\langle y^{n+1 / 2}\right\rangle & \text { for } n \text { odd. }\end{cases}
$$

Now let $k \geqq 0$ and let $Z_{k}$ denote the set

$$
\begin{aligned}
& Z_{k}=\left\{\left(n_{0}, n_{1}, \ldots, n_{k}\right) \in \mathbb{N}^{k+1} \mid n_{0}=1\right. \text { and, } \\
&\left.1 \leqq i \leqq k, \text { either } n_{i}=2 n_{i-1} \text { or } n_{i}=2 n_{i-1}-1\right\} .
\end{aligned}
$$

Define, for each $k \in \mathbb{N}$, a map $f_{k}: \mathbb{N} \rightarrow \mathbb{N}$ by $f_{1}(n)=2 n-1$ and $f_{k}(n)=2 f_{k-1}(n)-1$ for $k \geqq 2$. Finally, for $k \geqq 0$ and $N \in Z_{k}$, put

$$
\left(B_{N, k}\right)_{i}= \begin{cases}\left\langle y^{n_{i}}\right\rangle & \text { for } 0 \leqq i \leqq k, \\ \left\langle y^{f_{i-k}\left(n_{k}\right)}\right\rangle & \text { for } i \geqq k+1,\end{cases}
$$

where $N=\left(n_{0}, n_{1}, \ldots, n_{k}\right)$. 
From (1), $\left(\left(B_{N, k}\right)_{i}\right)_{i \geqq 0}$ is an $\alpha$-sequence of ideals of $R$ and, by the remark following Definition 2.3, each $\left(B_{N, k}\right)_{i}$ is closed. By Theorem 2.5, it therefore defines an ideal of $A(R, \alpha)$, which will be denoted by $B_{N, k}$. The collection $\left\{B_{N, k} \mid k \geqq 0, N \in Z_{k}\right\}$ of ideals of $A(R, \alpha)$ will be denoted by $X$. It is now claimed that, given $B_{N_{, k}}, B_{N_{1}, k_{1}} \in X$, with $B_{N, k} \subsetneq B_{N_{1}, k_{1}}$, there exists an infinite descending chain of ideals in $X$ between $B_{N, k}$ and $B_{N_{1}, k_{1}}$. Indeed, since $B_{N, k} \neq B_{N_{1}, k_{1}}$, by Theorem 2.5 , there exists $m \geqq k+k_{1}$ such that $\left(B_{N, k}\right)_{m} \subsetneq\left(B_{N_{1}, k_{1}}\right)_{m}$. Assume that $\left(B_{N, k}\right)_{m}=\left\langle y^{m_{0}}\right\rangle$ and $\left(B_{N_{1}, k_{1}}\right)_{m}=\left\langle y^{m_{1}}\right\rangle$. Since $m \geqq k+k_{1},\left(B_{N, k}\right)_{m+1}=\left\langle y^{2 m_{0}-1}\right\rangle$ and $\left(B_{N_{1}, k_{1}}\right)_{m+1}=\left\langle y^{2 m_{1}-1}\right\rangle$. Define

$$
\left(B_{N_{2}, k_{2}}\right)_{i}= \begin{cases}\left(B_{N_{1}, k_{1}}\right)_{i} & \text { for } 0 \leqq i \leqq m, \\ \left\langle y^{2 m_{1}}\right\rangle & \text { for } i=m+1, \\ \left\langle y^{f_{i-m-1}\left(2 m_{1}\right)}\right\rangle & \text { for } i \geqq m+2 ;\end{cases}
$$

so that $k_{2}=m+1$, and $N_{2} \in Z_{k_{2}}$ has $j$ th entry $n_{j}$ such that $\left(B_{N_{2}, k_{2}}\right)_{j}=\left\langle y^{n_{j}}\right\rangle$. Then, since $m_{0} \geqq m_{1}+1,2 m_{1}-1<2 m_{1}<2 m_{0}-1$, and $\left(B_{N, k}\right)_{m+1} \varsubsetneqq\left(B_{N_{2}, k_{2}}\right)_{m+1} \subsetneq\left(B_{N_{1}, k_{1}}\right)_{m+1}$. Also, since each $f_{k}$ is an increasing function, $\left(B_{N, k}\right)_{i} \subsetneq\left(B_{N_{2}, k_{2}}\right)_{i} \subsetneq\left(B_{N_{1}, k_{1}}\right)_{i}$ for all $i \geqq m+2$. Hence $B_{N, k} \subsetneq B_{N_{2}, k_{2}} \subsetneq B_{N_{1}, k_{1}}$. The process can be repeated for $B_{N, k} \subsetneq B_{N_{2}, k_{2}}$, and repeated application yields the required infinite descending chain.

Now assume that $A(R, \alpha)$ has Krull dimension. Then, by Lemma 1.1 of [2], the $A(R, \alpha)$-module $I / J$ has Krull dimension, for any ideals $I \supsetneqq J$ of $A(R, \alpha)$. Let $l, J \in X$ be such that $I \supsetneqq J$ and $\mathrm{K} \operatorname{dim} I / J=\min \{\mathrm{K} \operatorname{dim} A / B \mid A \supsetneqq B, A, B \in X\}$. As shown above, there exists an infinite descending chain $\left(I_{j}\right)_{j \geqq 0}$ of ideals in $X$ with $J \subsetneq I_{j} \subsetneq I$ for all $j \geqq 0$. By definition of Krull dimension, there must exist $k \geqq 0$ such that, for all $j \geqq k$, $\mathrm{K} \operatorname{dim}\left(I_{j} / I_{j+1}\right)<\mathrm{K} \operatorname{dim} I / J$. This is a contradiction; so $A(R, \alpha)$ cannot have Krull dimension.

5. Ascending chain condition on annihilator ideals. It has been shown [7, Corollary 2.23] that if $R$ has finite left Goldie dimension then so must $A(R, \alpha)$. It is natural, therefore, to ask whether the other Goldie condition, the ascending chain condition for annihilator left ideals, is passed from $R$ to $A(R, \alpha)$. The following example shows that this need not be the case-the ring $R$ concerned was first used by J. W. Kerr [5] as an example of a ring with acc on annihilators but no bound on the lengths of chains of annihilators.

EXAMPLE 5.1. Let $K$ be a field and let

$$
\hat{Y}=\left\{\hat{y}_{i j} \mid i, j \in \mathbb{N}, j \leqq i\right\}
$$

be a collection of commuting indeterminates. Let $\hat{\alpha}: K[\hat{Y}] \rightarrow K[\hat{Y}]$ be the $K$ monomorphism such that $\hat{\alpha}\left(\hat{y}_{i j}\right)=\hat{y}_{i+1, j+1}$, and consider the ideal $I$ of $K[\hat{Y}]$ generated by

$$
\left\{\hat{Y}^{3}, \hat{y}_{i j} \hat{y}_{i k} \mid i, j, k \in \mathbb{N}, k \neq j\right\} .
$$

It is clear that $\hat{\alpha}(I) \subseteq I$, and it can also be shown that $l$ is $\hat{\alpha}$-stable. Therefore, $\hat{\alpha}$ defines, 
in a natural way, a monomorphism

$$
\alpha: \frac{K[\hat{Y}]}{I} \rightarrow \frac{K[\hat{Y}]}{I} .
$$

The commutative ring $K[\hat{Y}] / I$ will be denoted by $R, Y$ will denote the image of $\hat{Y}$ in $R$, and $y_{i j}$ will denote the image of $\hat{y}_{i j}$ in $R$. It is shown by Kerr [5] that $R$ has acc on annihilator ideals.

Now, consider the ring $A(R, \alpha)$, and consider an element of the form $x^{-m} y_{m+1,1} x^{m}$. Then $\left(x^{-m} y_{m+1,1} x^{m}\right)^{2} \neq 0$; but, for $n \geqq 0$ with $n \neq m$,

$$
\begin{aligned}
\left(x^{-n} y_{n+1,1} x^{n}\right)\left(x^{-m} y_{m+1,1} x^{m}\right) & =x^{-(m+n)} \alpha^{m}\left(y_{n+1,1}\right) \alpha^{n}\left(y_{m+1,1}\right) x^{m+n} \\
& =x^{-(m+n)} y_{m+n+1, m+1} y_{m+n+1, n+1} x^{m+n} \\
& =0
\end{aligned}
$$

because of the definition of the ideal $l$.

Now let $B_{n}=\left\{x^{-m} y_{m+1,1} x^{m} \mid m \geqq n\right\}$ for each $n \geqq 0$. Since $B_{n} \supseteq B_{n+1}$ for all $n \geqq 0$, certainly $l\left(B_{n}\right) \subseteq l\left(B_{n+1}\right)$. But, from above, $x^{-n} y_{n+1,1} x^{n} \in l\left(B_{n+1}\right)$ but $x^{-n} y_{n+1,1} x^{n} \notin l\left(B_{n}\right)$. Thus $\left(l\left(B_{n}\right)\right)_{n \geqq 0}$ is an infinite ascending sequence of annihilators of $A(R, \alpha)$.

Remark. Although this example shows that acc on left annihilators need not be passed from $R$ to $A(R, \alpha)$, the ring $R$ has infinite Goldie dimension, and it is not known what happens if $R$ has finite Goldie dimension. In other words, it is not known whether $R$ being left Goldie forces $A(R, \alpha)$ also to be left Goldie.

ACKNowledgement. All the results presented in this paper appeared in the author's Ph.D. thesis [7], and I would like to take this opportunity to thank my Ph.D. supervisor, Dr C. R. Hajarnavis, for all his help during the preparation of my thesis.

\section{REFERENCES}

1. C. Dean, Monomorphisms and radicals of Noetherian rings, J. Algebra 99 (1986), 573-576.

2. R. Gordon and J. C. Robson, Krull dimension, Memoirs of the American Mathematical Society 133 (1973).

3. A. V. Jategaonkar, Skew polynomial rings over orders in Artinian rings, J. Algebra 21 (1972), 51-59.

4. D. A. Jordan, Bijective extensions of injective ring endomorphisms, J. London Math. Soc. (2) 35 (1982), 435-448.

5. J. W. Kerr, Ph.D. Thesis, University of California, San Diego (1979).

6. R. B. Warfield, Bezout rings and serial rings, Comm. Algebra, 7 (1979), 533-545.

7. J. C. Wilkinson, Ph.D. thesis, University of Warwick (1983).

8 Westenra Terrace

CASHMERE

CHRISTCHURCH 2

New Zealand 